\title{
Blue light protecting cosmetic active ingredients: a case report
}

\begin{abstract}
Background: High energy visible lights more and more attracts notice in the cosmetic science field. It is well known that UV light harms skin cells and unprotected exposure to this harmful radiation leads to premature skin ageing. Recently, the impact of blue light on human skin is investigated in-vitro and in-vivo. Evidence grows that blue light increases reactive oxygen species (ROS) intracellular which leads to cell damage and subsequent apoptosis.
\end{abstract}

Methods: HaCaT cells were cultivated and incubated with anti-oxidative cosmetic active ingredients. After irradiation of the cells with defined blue light, the intracellular ROS were detected by fluorescent staining and cell sorting. The effect of one cosmetic active ingredient was determined in an application study for three months, visualization of rosacea teleangiectoides and quantification of red spots was done with a VISIA-CR photographic system.

Results: All investigated cosmetic active ingredients exhibited ROS reduction close to the baseline level after 2 hours of blue light irradiation. Application of one cosmetic active ingredient to the facial skin revealed a statistically significant reduction in red spots count and a markedly reduction of rosacea teleangiectoides by $44.3 \%$ in one test subject.

Conclusion: Anti-oxidant cosmetic active ingredients are capable of protecting skin cells from the impact of blue light and lead to a visible reduction of ROS induced skin aberrations in vivo.

Keywords: radiation, cytotoxicity, glycerin, water, levan, decyl glucoside, olea europaea, leaf extract, phenethyl alcohol, zizyphus
Volume I Issue 4 - 2017

\author{
Stefan Hettwer, Emina Besic Gyenge, Barbara \\ Obermayer
}

Senior R\&D Manager Cosmetic Actives, Switzerland

Correspondence: Stefan Hettwer, Rahn AG, Dörflistrasse 120, 8050 Zürich, Switzerland, Tel +4I 4431542 00,

Email stefan.hettwer@rahn-group.com

Received: November 16, 2017 | Published: December 12, 2017

\section{Introduction}

The sun is a powerful light source emitting radiation in all kind of wavelengths from the gamma-rays up to the long radio-waves. The visible light is only a tiny part of the spectrum but is most important to us human beings. In proximity to the visible light, UV-A, B and C radiation is the biggest thread for living organisms. This energy rich UV radiation is able to destroy molecular bonds and damage DNA, proteins and lipids. ${ }^{1}$ In the early days of evolution, life was most likely shielded from sunlight. One suggestion locates the origin of life in the deep sea at hydrothermal vents, mineral rich hot volcanic sources at the sea-bed. ${ }^{2}$ The first ancient microbes generated their metabolic energy from consumption of hydrogen and sulfur compounds. ${ }^{3}$ Settlement at the upper region of the ocean required mechanisms to cope with energy rich UV-A, B and C radiation. As such, effective DNA repair mechanisms were evolved. An additional threat was the development of photosynthesis. This photo-chemical process uses inorganic $\mathrm{CO}_{2}$ and water to produce energy rich glucose. As a waste product, air-oxygen is released. This is a very reactive oxidative agent, which is, at the first glance a toxic compound to the cells. As a protective measure, oxidative stress enzymes like superoxide dismutase, catalase and glutathione reductase were evolved, just to mention a subset of reactive oxygen species (ROS) eliminating enzymes. ${ }^{4}$ Over millions of years, cyanobacteria and the first algae produced so much oxygen that we now have more than $20 \%$ of it in our atmosphere. This oxygen facilitated the evolution of higher life on earth. On the one hand, the respiration of oxygen could be used to generate metabolic energy, on the other hand, air oxygen facilitates the generation of ozone in the atmosphere, which absorbs all UV-C and most of the UV-B radiation. ${ }^{5}$ From that point in time, life on the earth's surface was possible without dangerous radiation destroying all relevant molecules important for metabolism and reproduction.

Nonetheless, the residual amount of UV-radiation is still very harmful to us. We can recognize this by getting sunburn when we expose ourselves for too long to the sunlight. The skin pigment melanin and of course sun protection factors in cosmetic products are meant to protect us against UV-radiation. But there is also a high energy portion of light close to the UV-spectrum in the visible fraction of light: Blue light or high energy visible light (HEV). Recent studies indicate that exposure to blue light emitting LED displays as can be found in televisions, and smart devices, including eBook readers, can lead to substantial insomnia as the production of the sleeping hormone melatonin is suppressed. ${ }^{6}$ Opposite, blue light is sometimes used to cure certain skin abnormalities like acne ${ }^{7}$ or psoriasis. ${ }^{8}$ This form of therapy takes advantage of naturally occurring molecules in the skin which absorb blue light. As a matter of fact, these so called photosensitizers are lifted up onto a radical like excitation level. By that, they can do harm to aberrant cells, which eventually die. Of course, also healthy cells are affected but they have either the capability to regenerate or are rapidly replaced. Nonetheless, a potential harmful effect on the skin due to blue light exposure is being more and more discussed and recent publications make clear that excessive blue light exposure leads to skin cell aberrations and apoptosis. ${ }^{9}$ 
The reason of cytotoxicity of blue light to cells is the generation of intracellular ROS. These blue light induced ROS can already be detected by irradiating the cells with a moderate dose of $13 \mathrm{~J} / \mathrm{cm}^{2}$ (see results section). For comparison, the UV-dose in spring at sea level varies between $100-180 \mathrm{~J} / \mathrm{cm}^{2} .^{10}$ Three different cosmetic actives with anti-oxidant capacity were tested for their ability to reduce blue light induced ROS accumulation in keratinocytes. As an elevated ROS level can lead to premature skin ageing ${ }^{11}$ and rosacea, ${ }^{12}$ we were interested how cosmetic active ingredients can interact with the human skin.

\section{Materials and methods}

\section{Cosmetic active ingredients}

Three different cosmetic active ingredients were selected due to their anti-oxidative capacity. CELLIGENT ${ }^{\circledR}$ (INCI: Helianthus Annuus (Sunflower) Seed Oil, Ethyl Ferulate, Polyglyceryl5Trioleate, Rosmarinus officinal's(Rosemary) Leaf Extract, Water, Disodium Uridine Phosphate, Tocopherol) contains carnosolic acid from rosemary and ethyl ferulate as ROS scavenging molecules. MYRAMAZE $^{\circledR}$ (INCI: Propanediol, Water, Myrothamnus Flabellifolia Extract, Ascorbic Acid, Citric Acid) is rich in O-galloylquinic acids and PROTEOLEA ${ }^{\circledR}$ (INCI:Glycerin, Water, Levan, Decyl Glucoside, Olea Europaea (Olive) Leaf Extract, Phenethyl Alcohol, Zizyphus Jujuba Seed Extract, Citric Acid, Ascorbic Acid)contains oleuropein. The amount of anti-oxidant molecules varies between $0.5 \%$ and $4 \%$.

\section{Determination of ROS in human keratinocytes}

The human keratinocyte cell line $\mathrm{HaCaT}$ was suspension-cultivated in DMEM medium supplemented with 10\% FCS. 100000cells/well were transferred into 24 well plates. Cells were incubated with or without cosmetic active ingredient at $0.05 \%$ or $0.01 \%$ for 2 hours Subsequently, the cells were irradiated with a $270 \mathrm{~mW}$ blue LED light $\left(\lambda_{\max }=470 \mathrm{~nm}\right)$ for 2 hours. The emitted energy was $13 \mathrm{~J} / \mathrm{cm}^{2}$ according to $1.8 \mathrm{~mW} / \mathrm{cm}^{2}$ and $120 \mathrm{~min}$ exposure time. For the determination of ROS-positive cells, the non-fluorescent dye 5(6)-carboxy-2',7'dichlorofluorescein diacetate (DFFH-DA) was added to the treated cells. In case of elevated oxidative stress, the acetate moiety is cleaved off, resulting in the fluorescent dye 5(6)-carboxy-2',7'dichlorofluorescein. Cells were washed with ice-cold phosphate buffered saline (PBS), supplemented with $10 \mu \mathrm{M}$ DFFH-DA and incubated for $30 \mathrm{~min}$ at $37^{\circ} \mathrm{C}$ at $5 \% \mathrm{CO}_{2}$. After washing with PBS, cell fluorescence was assessed by flow cytometry (Cytomics FC500MCL).

\section{In-Vivo study}

The study was performed in accordance with the principles of good laboratory practice (GLP) and good clinical practice (GCP) and in compliance with the quality assurance system requirements. Studies were conducted with respect to World Medical Association in the Declaration of Helsinki. All study participants signed a written informed consent at the beginning of the study.

Double blind, placebo-controlled study. 21 female subjects aged 35-65 with healthy, Caucasian skin applied a placebo formulation (INCI: Water, Caprylic/Capric Triglyceride, CetearylIsononanoate, Diethylhexyl Carbonate, Sucrose Distearate, Glycerin, Cetearyl Alcohol, Sucrose Stearate, Phenoxyethanol, Carbomer, Sodium Stearoyl Glutamate, Xanthan Gum, Caprylyl Glycol, Sodium
Hydroxide) twice daily for 3 months. Another 32 volunteers applied the identical formulation containing 3\% MYRAMAZE ${ }^{\circledR}$ (INCI: see above) in the same way.

Skin redness and appearance of rosacea teleangiectoides were investigated by VISIA-CR (Canfield, USA) photography in the visual and $\mathrm{RBX}^{\circledR}$ red channel at day 0, day 29 and day 84. Quantification of red spot count was done with the integrated software of the device.

\section{Results}

\section{Oxidative stress in keratinocytes}

In the untreated $\mathrm{HaCaT}$ cell population, $32.4 \%$ of the cells were ROS positive as deduced from the generation of 5(6)-carboxy$2^{\prime}, 7^{\prime}$-dichlorofluorescein after addition of 5(6)-carboxy-2', $7^{\prime}$ dichlorofluorescein diacetate. The irradiation of $\mathrm{HaCaT}$ cells with a $240 \mathrm{~mW}$ LED lamp $\left(\lambda_{\max }=470 \mathrm{~nm} ; 13 \mathrm{~J} / \mathrm{cm}^{2}\right)$ for 2 hours lead to a significant increase of 6-carboxy-2',7'-dichlorodihydrofluorescein positive cells. After blue light irradiation irradiation, $59.7 \%$ of the cells were ROS positive, corresponding to an increase of $84 \%$. Upfront incubation for 2 hours with CELLIGENT ${ }^{\circledR}$ at $0.05 \%$, MYRAMAZE ${ }^{\circledR}$ at $0.01 \%$ and PROTEOLEA ${ }^{\circledR}$ at $0.01 \%$ significantly reduced the ROS positive fraction of blue-light irradiated cells close to the basal level (Table $1 \&$ Figure 1).

Table I Fraction of cells positive for fluorescent 5(6)-Carboxy-2', 7'-dichlorofluorescein

\begin{tabular}{ll}
\hline & Fraction of ROS positive cells $(\% ;$ mean \pm SD) \\
\hline Untreated & $32.4 \pm 5.2$ \\
Control (blue light) & $59.7 \pm 11.3$ \\
CE $0.05 \%$ & $37.8 \pm 6.6$ \\
MY $0.01 \%$ & $37.8 \pm 7.8$ \\
PR $0.01 \%$ & $34.2 \pm 6.0$ \\
\hline
\end{tabular}

\section{Intracellular ROS accumulation}

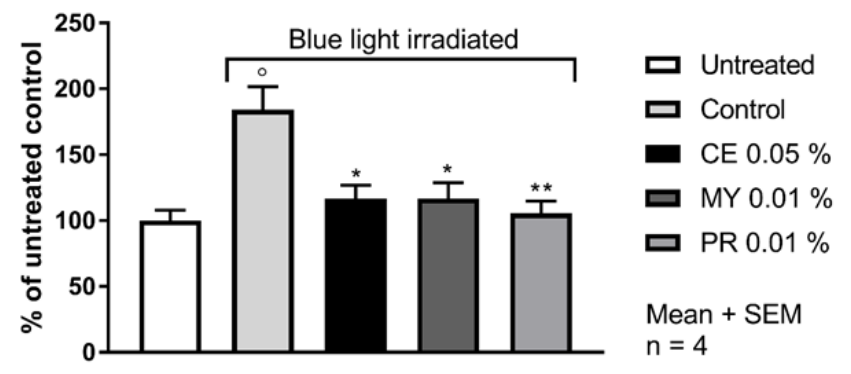

Figure I ROS accumulation in $\mathrm{HaCaT}$ cells upon blue light irradiation Cells were incubated for 2 hours with or without supplemented cosmetic ingredient and irradiated at $13 \mathrm{~J} / \mathrm{cm} 2$ with blue light $(\lambda \max =470 \mathrm{~nm})$. After labelling with 5(6)-Carboxy-2',7'-dichlorofluorescein diacetate, fluorescent cells were counted by cell sorting. CE: CELLIGENT®; MY: MYRAMAZE®; PR PROTEOLEA®. ${ }^{\circ} \mathrm{p}<0.05$ compared to untreated; * $\mathrm{p}<0.05$ compared to blue light control; $*^{*} \mathrm{p}<0.0$ I compared to blue light control. Unpaired Student's t-test. 


\section{Potential benefit in people suffering from rosacea}

Reduction of rosacea was investigated in an application test on 53 women ( 21 placebo, 32 with $3 \%$ MYRAMAZE $^{\circledR}$ ). The test products were applied twice daily on the face for 84 days. Rosacea teleangiectoides was markedly reduced in single study participants. In average, red spots were significantly reduced by $8.5 \%$ in 84 days over baseline and placebo (unpaired Student's t-test). The maximal reached improvement was observed in study participant \# 21 with a $44.3 \%$ reduction of red spots, mainly caused by capillaries (Figure 2 ).

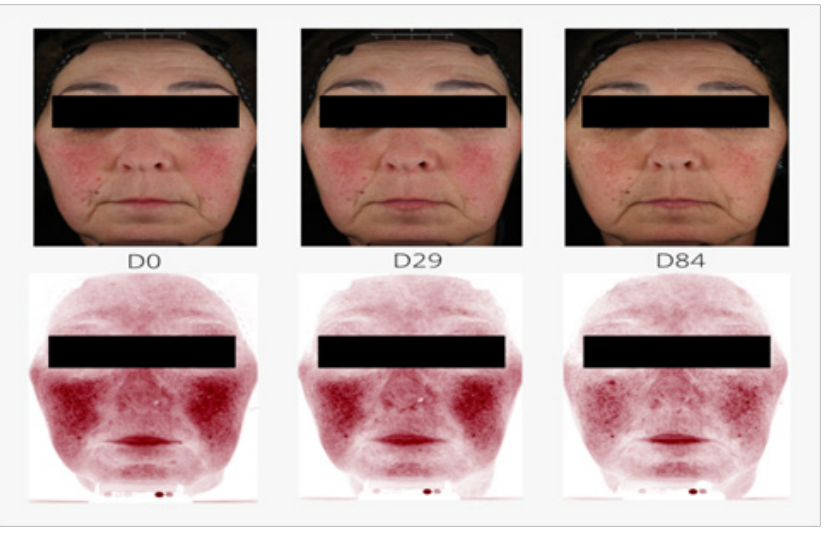

Figure 2 Reduction of rosacea teleangiectoides in a study participant (\#2I; female, Caucasian skin, aged 58 years). During application of $3 \%$ MYRAMAZE®, the pronounced rosacea (D0) is subsequently reduced until day 84(D84). Top panels: real color images. Bottom panels: RBX®red channel of the VISIA-CR device.

\section{Discussion}

Blue light is an underestimated threat leading to elevated ROS in epithelial tissue..$^{13}$ Although cosmetic formulations exist effectively shielding UV-A and UV-B from the skin, a blue light filter may be hard to develop. The reason is not the lack of molecules absorbing in the blue light region but rather the acceptance of the consumer. While chemical UV absorbers are generally colorless, blue light absorbers with sufficient light protection factors would be bright yellow or orange on the skin, which is inacceptable for the use as a cosmetic. As such, only the amelioration of effects caused by blue light impact can be achieved with blue light protection cosmetic active ingredients. The main impact is the generation of intracellular ROS as a consequence of shifting blue light absorbing molecules (photosensitizers) into an excited state. ${ }^{14}$ Together with intracellular oxygen, these excited molecules cause the generation of deleterious reactive species, which are harmful to DNA, proteins and lipids. A cosmetic ingredient meant to protect cells from such kind of damages has to be able to reduce the additional ROS load generated by blue light irradiation. All three cosmetic ingredients tested here were able to reduce the additional ROS load in HaCaT cells almost to the basal level, in concentration ranges between $0.01 \%$ and $0.05 \%$. As all three actives contain antioxidative molecules, they most likely support the cells to reduce the ROS generated by blue light irradiation or suppress the basal level of ROS which are generally appearing intracellular. Previous studies have shown the anti-oxidative potential of the actives in non-irradiated keratinocytes (data not shown). CELLIGENT ${ }^{\circledR}$ contains carnosolic acid from rosemary extract and ethyl ferulate. MYRAMAZE ${ }^{\circledR}$ is rich in O-galloylquinic acids from Myrothamnus flabellifolia and PROTEOLEA $^{\circledR}$ contains oleuropein from olive leaf extract. All these molecules belong to the most powerful natural anti-oxidants. The concentration range in the active ingredients varies between $0.5 \%$ and $4 \%$. Obviously, this concentration is enough to reduce the elevated ROS load of blue-light irradiated cells. Furthermore, an ameliorating effect of study participants suffering from rosacea teleangiectoides could be observed. Rosacea teleangiectoides is believed to be provoked by elevated ROS load of the skin. This condition leads to an inflammatory state with increased microcirculation and the formation and widening of small blood vessels, giving rise to the red skin color especially in the malar area. The exposition of the skin with blue light might aggravate this situation by generation of additional ROS. As such, the supplementation of the skin with anti-oxidants may reduce the ROS content in the skin and reduces skin redness and rosacea.

\section{Acknowledgements}

We like to acknowledge Zurko Bioresearch for the conduction of ROS measurement and $\mathrm{PhD}$ Trials for the conduction of the in-vivo study.

\section{Conflict of interest}

All authors are employees of RAHN AG.

\section{References}

1. De Jager TL, Cockrell AE, Du Plessis SS. Ultraviolet light induced generation of reactive oxygen species. Adv Exp Med Biol. 2017;996(2):1523.

2. Herschy B, Whicher A, Camprubi E, et al. An origin-of-life reactor to simulate alkaline hydrothermal vents. J Mol Evol. 2014;79:213-227.

3. Stewart LC, Llewellyn JG, Butterfield DA, et al. Hydrogen and thiosulfate limits for growth of a thermophilic, autotrophic Desulfurobacterium species from a deep-sea hydrothermal vent. Environ Microbiol Rep. 2016;8(2):196-200.

4. Sorg O. Oxidative stress: a theoretical model or a biological reality? $C R$ Biol. 2004;327(7):649-662.

5. Kripke ML. Impact of ozone depletion on skin cancers. J Dermatol Surg Oncol. 1988;14(8):853-857.

6. Tosini G, Ferguson I, Tsubota K. Effects of blue light on the circadian system and eye physiology. Mol Vis. 2016;22:61-72.

7. Morton CA, Scholefield RD, Whitehurst C, et al. An open study to determine the efficacy of blue light in the treatment of mild to moderate acne. J Dermatolog Treat. 2005;16(4):219-223.

8. leinpenning MM, Otero ME, van Erp PE, et al. Efficacy of blue light vs. red light in the treatment of psoriasis: a double-blind, randomized comparative study. J Eur Acad Dermatol Venereol. 2012;26:219-225.

9. Bennet D, Viswanath B, Kim S, et al. An ultra-sensitive biophysical risk assessment of light effect on skin cells. Oncotarget. 2017;8(29):4786147875 .

10. Marionnet C, Tricaud C, Bernerd F. Exposure to non-extreme solar UV daylight: spectral characterization, effects on skin and photoprotection. Int J Mol Sci. 2014;16(1):68-90.

11. Birch Machin MA, Bowman A. Oxidative stress and ageing. Br J Dermatol. 2016;175(2):26-29. 
12. Jones D. Reactive oxygen species and rosacea. Cutis. 2004;74(3):1720,32-34.

13. Lee JB, Kim SH, Lee SC, et al. Blue light-induced oxidative stress in human corneal epithelial cells: protective effects of ethanol extracts of various medicinal plant mixtures. Invest Ophthalmol Vis Sci. 2014;55(7):41194127.
14. Rozanowska M, Wessels J, Boulton M, et al. Blue light-induced singlet oxygen generation by retinal lipofuscin in non-polar media. Free Radic Biol Med. 1998;24:1107-1112. 